\title{
Prevalence and Determinants of Readiness of Health Facilities for Quality Antenatal Care Services in Bangladesh
}

Shahnaz Nilima ( $\sim$ s.nilima@du.ac.bd)

University of Dhaka

Kanchan Kumar Sen

University of Dhaka

Fatima Tuz Zahura

University of Dhaka

Wasimul Bari

University of Dhaka

\section{Research Article}

Keywords: Readiness, Antenatal Care, Health Facility, Multinomial Regression, Bangladesh

Posted Date: April 6th, 2021

DOI: https://doi.org/10.21203/rs.3.rs-376595/v1

License: (c) (i) This work is licensed under a Creative Commons Attribution 4.0 International License.

Read Full License 


\section{Title}

Prevalence and determinants of readiness of health facilities for quality antenatal care services in Bangladesh

\section{Authors}

1. Shahnaz Nilima

(Corresponding author)

Email: $\underline{\text { s.nilima@du.ac.bd }}$

2. Kanchan Kumar Sen

Email: kksen.sta@du.ac.bd

3. Fatima Tuz Zahura

Email: zahura.fatima@yahoo.com

4. Wasimul Bari

Email: $\underline{\text { w_bari@du.ac.bd }}$

Address $^{1-4}$ : Department of Statistics, University of Dhaka, Dhaka-1000, Bangladesh 


\section{Abstract}

\section{Background}

3 The quality antenatal care (ANC) services can reduce the risk of the pregnancy complications, and

4 hence reduce the maternal and child morbidity and mortality. To ensure the quality ANC services

5 to the pregnant women, it is essential that healthcare providers should be fully prepared with six

6 tracer indicators recommended by World Health Organization. In this study, the prevalence of

7 readiness by selected covariates has been examined. Potential factors responsible for the readiness

8 have also been identified.

\section{Methods}

10 Using data from nationally representative Bangladesh Health Facility Survey (BHFS), 2017, the 11 readiness indices of health facilities providing ANC services have been measured based on the six

12 tracer indicators of the service. The chi-square test has been applied to check the association of 13 selected covariates with the readiness index, and to obtain the adjusted associations of covariates, 14 we have carried out a multinomial logistic regression model.

\section{Results}

16 Only $4.26 \%$ of the facilities is found to provide quality ANC services to the pregnant women.

17 Rural facilities have lower readiness to provide quality services compared to urban facilities [RRR: 0.13, 95\% CI: 0.06-0.31; $\mathrm{p}<0.001]$. Community clinics and private hospitals are less likely to have

19 medium or high readiness compared to public hospitals or clinics. The health facilities having 20 specialist or MBBS doctors are more likely to be considered as ready for quality ANC services 21 compared to others facilities. Regional difference exists in readiness for providing the service. 


\section{Conclusion}

23 A huge gap has been found in the facilities of Bangladesh to prove quality ANC services. This is

24 a high time to reduce this gap in achieving sustainable development goals related with maternity

25 and neonatal mortality. The present study recommends that the government of Bangladesh should

26 take necessary initiatives to fully prepared healthcare providers so that quality ANC services can

27 be equally provided to each pregnant woman.

28 Keywords: Readiness, Antenatal Care, Health Facility, Multinomial Regression, Bangladesh

29

30

31

32

33

34

35

36

37

38

39

40 


\section{Background}

42 Antenatal care (ANC) is a widely accepted accessible and cost-efficient way to improve maternal

43 and perinatal health outcomes [1]. ANC links women with health system and helps them to get

44 proper information and advice regarding pregnancy complications, postnatal care including

45 newborn care, promotion of early and exclusive breastfeeding, and planned birth spacing [2-4].

46 Therefore, negative pregnancy outcomes can be impeded by identifying and treating maternal

47 health compilations during pregnancy through ANC [2]. Better quality antenatal care can promote

48 a woman to choose skilled care for herself and her child at and after birth of the child [5-8].

49 Furthermore, ANC could prevent maternal and neonatal deaths through prevention, treatment

50 during pregnancy and also skilled care at and after childbirth [9]. Reducing maternal mortality

51 ratio $(\mathrm{MMR})$ as well as neonatal mortality rate (NMR) is considered as one of the global key

52 priorities. In order to ensure this, the international community has adopted the third sustainable

53 development goal (SDG 3) which aims to reduce MMR and NMR to 70 per 100000 live births and

5412 per 1000 live births, respectively by 2030 [10]. Despite some achievements, around 295000

55 maternal deaths and 2.4 million neonatal deaths occur worldwide $[10,11]$. Low resource countries

56 account for majority of these deaths and most could be prevented if women had access to antenatal

57 care [11-13]. As the reason for most maternal deaths is pregnancy or childbirth related

58 complications, quality ANC could reduce such complications and hence results in lower maternal

59 mortality [12]. Again, the risk of morbidity and mortality of their children can also be reduced by

60 ensuring adequate maternity care during pregnancy [14].

Although four ANC visits during pregnancy are required to ensure the safety of mothers and

62 their children, World Health Organization (WHO) recently revised ANC guidelines for most

63 efficacious pregnancy outcomes $[4,15]$. According to new guidelines, a minimum of eight ANC 
64 visits in the absence of complications is recommended throughout pregnancy period, which will

65 be challenging for developing countries to achieve this target due to many barriers [15]. Moreover,

66 in order to meet this challenge, health facilities need to be well prepared with trained staffs,

67 medicines, equipment, and infrastructure [8]. The availability and readiness to offer the services

68 are important determinants of health care service utilization [8]. Increasing ANC utilization is

69 essential to improve maternal and newborn health outcomes, but it fails to meet the targets of SDG

703 if quality ANC offered by health facilities is poor [16, 17]. Availability of services is deemed

71 as a primary requirement for high-quality care delivery: and investigating readiness of healthcare

72 providers is critical for understanding quality of care [16]. Therefore, examining readiness to offer

73 ANC is required to improve quality of ANC.

Although some progress has been made over the last two decades, Bangladesh is far away from achieving SDG 3 by 2030 [18]. The Health, Population and Nutrition Sector Development

76 Program (HPNSDP) has set a target that 50 percent of pregnant women should complete at least

77 four ANC visits by 2022 in Bangladesh [2]. The Bangladesh Demographic and Health Survey 78 (BDHS), 2017 showed that 82 percent of women received ANC from a skilled provider, while 79 only 47 percent had completed four or more ANC visits during the last pregnancy [18]. Lack of 80 access to health facilities and skilled health providers are responsible to refrain women from 81 receiving four or more ANC visits during pregnancy in Bangladesh [9]. Previous studies showed 82 that women receiving ANC late in the pregnancy reported poor experiences because of inadequate 83 time for counselling and mistreatment from providers [19, 20]. Furthermore, maternal as well as 84 neonatal mortality and morbidity can be reduced by assuring quality antenatal care services 85 through high readiness of health care facilities $[19,21]$. 
To provide information on health system functioning and readiness of health care facility

87 services, Service Provision Assessment (SPA) or Health Facility Survey (HFS) has been conducted

88 in developing countries. A number of HFSs has also been conducted in Bangladesh to assess

89 availability and readiness of several facility-based health care services such as child health,

90 antenatal care and newborn care, family planning (FP) as well as services for selected non-

91 communicable diseases (diabetes, cardiovascular diseases) and tuberculosis. The Bangladesh HFS

92 (BHFS), 2017 reported that almost all (99\%) health facilities offer antenatal care services in

93 Bangladesh, whereas only $4 \%$ are ready to provide quality ANC services according to World

94 Health Organization (WHO) criteria [2]. To confirm better quality antenatal care services at health

95 facilities, the huge difference between the availability and the readiness of these services needs to

96 be reduced.

Although several studies have been conducted in Bangladesh to explore readiness of

98 healthcare facilities to provide general health services [22], non-communicable diseases [23-27],

99 child curative care [28] and child immunization services [29], readiness to provide quality ANC is

100 still unexplored. So, this study has made first attempt to investigate on readiness of antenatal care

101 services in Bangladesh. The purpose of the study is to find out the prevalence and influential

102 factors of readiness for providing quality ANC services in Bangladesh. For this purpose, BHFS, 1032017 data has been utilized.

\section{Methods}

\section{Data}

106 This study utilized data derived from nationally representative Bangladesh Health Facility Survey 107 (BHFS), 2017. The BHFS, 2017 was based on service provision assessment (SPA) and conducted 
108 in Bangladesh under the authority of National Institute of Population Research and Training 109 (NIPORT) of Ministry of Health and Family Welfare (MOHFW) with financial assistance of 110 Government of Bangladesh and U.S. Agency for International Development (USAID). The sample 111 of 2017 BHFS was a stratified random sample of 1600 health facilities selected from all registered 112 health facilities across all eight administrative divisions in Bangladesh. Finally, interviewers 113 successfully collected information from 1524 health facilities.

114 In this study, in order to assess the readiness of antenatal care services, the facilities which offer 115 antenatal care services were selected. Among the interviewed facilities, 1505 facilities were 116 reported available to provide ANC services and those facilities were taken in this study. Again, 117 data have been weighted i.e., complex survey design has been used in the study in order to avoid 118 overestimation or underestimation problem.

\section{Outcome Variable}

120 We used service availability and readiness assessment (SARA) reference manual of WHO to 121 identify the items or tracer indicators that a facility needs to offer quality ANC services [30]. To 122 define the readiness of facilities providing antenatal care, less restrictive as well as Bangladesh 123 context-oriented version of ANC service readiness measure has been applied in this study [2]. To 124 serve this purpose, six tracer indicators under five domains given in Table 1 have been used.

128 In the study, outcome variable was readiness of health facilities to offer antenatal care services. 129 The readiness index was created by WHO approach, where equal weights were given to each 
130 domain of indicators/ items and also to each item at each weighted domain for providing antenatal 131 care services. The above six items are considered for each facility and summed up to generate 132 weighted mean readiness score $[8,28,30,31]$. Finally, mean score was multiplied by 100 to obtain 133 the results of readiness score in percentage. Mathematically, the readiness score for the $i^{\text {th }}$ facility 134 can be expressed as follows [28]

$$
Y_{i}=\left(\frac{1}{m} \sum_{j=1}^{m} \frac{1}{p_{j}} \sum_{k=1}^{p_{j}} z_{i j k}\right) \times 100 ; i=1,2, \ldots, n ; j=1,2, \ldots, m ; k=1,2, \ldots, p_{j}
$$

138 and $n$ is the total number of facilities. This readiness index was a continuous variable giving value 139 between 0 and 100. The higher value of readiness score indicates higher ANC service readiness of 140 the health facilities whereas lower value implies lower readiness.

142 study. The facilities with readiness score 75 or below are considered as having low readiness; 143 facilities with 75 to 99 score considered as having moderate readiness; and facilities with 100 score 144 considered to be highly ready (complete readiness) to offer antenatal care services. Thus, the 145 readiness score was categorized as low, medium and high readiness in this study.

\section{Covariates}

147 In the study, based on the previous studies on readiness of health facilities, the six covariates were 148 selected to explore how these are associated with the readiness of facilities to provide antenatal 149 care services $[23,28,32,33]$. The covariates are: location of facility (Urban and Rural), type of 150 facility (Public hospitals or clinics, Community Clinics, Non-Governmental Organization (NGO) 
151 hospital or clinics and Private hospitals), health provider status (Assigned and Not assigned), 152 qualification of health provider (Specialist or MBBS Doctors and Others), basic amenities in 153 facility (Inadequate, Moderate and Adequate amenities) and division (Barisal, Chattogram, Dhaka, 154 Khulna, Mymensingh, Rajshahi, Rangpur and Sylhet). The providers who are posted in a facility 155 are defined as the assigned provider, and who are hired or seconded/deputed in the facility are 156 defined as not assigned provider in the study. The variable "basic amenities" was created from 157 observed information on nine amenities which are 1. national electricity grid availability, 2. regular 158 electricity, 3. improved water source, 4. visual and auditory privacy, 5. client latrine, 6. 159 communication equipment, 7. computer with internet 8. emergency transport, and 9. separate 160 latrine or toilet for female clients. Based on the availability of the amenities, each of these 161 amenities was created as indicator variables (Yes/No). In this study, the principal component (PC) 162 analysis technique has been used to obtain a score index of basic amenities from nine indicator 163 variables for reducing dimensionality. Higher score of a facility as compared with any other 164 facilities indicates that the facility has more amenities than other facilities. The score index is 165 continuous variable and also named as basic amenities in facility in this study. The distribution of 166 scores was divided into three equal segments based on quantiles for study purpose. The lower part, 167 middle part and upper part of score index were named as inadequate, moderate and adequate 168 amenities, respectively.

169 Statistical analysis

170 To obtain the descriptive statistics for the selected variables, percentage frequency was computed.

171 To check associations between several covariates and readiness of antenatal care services, the chi172 square test was used in the study. Since readiness of antenatal care service is a polytomous outcome 173 variable, multinomial logistic regression model was used to determine adjusted associations of 
174 covariates with readiness of facilities [34]. STATA 14 package has been used in the study for 175 analyzing the data.

\section{Results}

177 Results obtained from univariate and bivariate analyses have been reported in Table 2. Table 3 178 represents the results of regression model.

\section{Univariate Results}

180 Table 2 reveals among 1505 health facilities offering ANC, only 4\% are completely ready to 181 provide ANC services in Bangladesh, while about $73 \%$ facilities have lower readiness. More than 182 half of health facilities giving ANC (55\%) have at least one staff who have in-service training for 183 ANC, while fewer than half of health facilities (46\%) follow ANC guidelines. Majority of health 184 facilities have reported the availability of blood pressure apparatus and necessary medicines (iron 185 and/or folic acid tablets), whereas only fewer (14\%) have confirmed the availability of functioning 186 equipment and reagents required for diagnostic test (hemoglobin and urine protein test) at the 187 facility. Most of facilities offering antenatal care services are from rural areas (about 93\%). 188 Among the facilities, approximately $67 \%$ of interviewed facilities are community clinics, whereas 189 only $3 \%$ and $4 \%$ are private hospitals and NGO hospital or clinics, respectively. More than nine 190 in ten of health facilities (98\%) have assigned health providers. Again, only $8 \%$ of facilities 191 offering antenatal care services have health professionals who are specialists or MBBS doctors. 192 The distribution of facilities having inadequate, moderate and adequate basic amenities are almost 193 similar. Approximately $20 \%$ and $19 \%$ of facilities offering antenatal care services situated in 194 Dhaka and Chattogram respectively, as compared with only 6\% and 7\% of those were from Sylhet 195 and Barisal respectively. 


\section{Bivariate Results}

197

198

199

200

201

202

203

204

205

206

207

208

209

210

211

212

213

214

215

216

217

218

\section{Overall Readiness}

From Table 2, it is observed that all the selected covariates except health provider status have significant associations with readiness of facilities to offer antenatal care services. About $23 \%$ of urban facilities are completely ready to provide antenatal care services, as compared with $3 \%$ of rural facilities. Again, approximately $45 \%$ and $31 \%$ of facilities located in urban areas have low and medium readiness of antenatal care services, respectively, whereas $75 \%$ and $23 \%$ of rural facilities are observed to have low and moderate readiness respectively. Among public hospitals or clinics, about $9 \%$ facilities have full readiness, while about $64 \%$ facilities have poor readiness to provide such services. Approximately eight in ten of the community clinics have lower readiness, whereas only $1 \%$ of community clinics are completely ready to ensure antenatal care services to their clients. More than half of NGO hospitals or clinics (56\%) are moderately ready to give the services, whereas fewer the one-fourth of those facilities have complete (23\%) and lower $(22 \%)$ readiness, respectively. Again, most of private hospitals (84\%) have low readiness as compared with $14 \%$ and $2 \%$ of private hospitals with moderate and full readiness of providing the services, respectively. About $2 \%$ of facilities not having assigned health providers have complete readiness, whereas $4 \%$ of those having assigned health providers are fully ready to give the services on antenatal care. Table 2 demonstrates that facilities who have specialist or MBBS doctors as health providers $(17 \%)$ are more likely to ensure highly ready service for antenatal care than those who have other health professionals (3\%). As basic amenities index increases, the low readiness of antenatal care facility services also decreases from $82 \%$ of facilities with inadequate amenities to $66 \%$ of those with adequate amenities. By division, the percentage of facilities with high readiness to offer antenatal care varies from a low of $2 \%$ in Rangpur to a high of $7 \%$ in Sylthet. 


\section{Domains (Tracer indicators) of Readiness}

220 Readiness to provide quality ANC services is measured based on five domains recommended by

221 WHO, which are: trained staff for ANC at any time, guidelines on ANC, equipment (blood

222 pressure apparatus), diagnostic capacity (hemoglobin and urine protein test) and medicines (iron

223 and/or folic acid tablets). Table 2 shows that among five domains, availability of medicines (iron

224 and/or folic acid tablets) to offer better ANC is highest (93\%), whereas only 14\% of health

225 facilities have reported necessary functioning equipment and reagents for diagnostic test

226 (hemoglobin and urine protein test) available at the facility.

(The Table 2 here)

Trained Staff for ANC at any time

230 Approximately 55\% of health facilities offering ANC services have at least one staff who have in231 service training on ANC. All covariates except type of facility and division exhibit no significant 232 association with training on ANC for at least one staff member of the facility. Among four types 233 of facilities, NGO hospitals or clinics are most likely to have at least one staff who received training 234 for ANC (64\%), whereas private hospitals are least likely to have trained staff for ANC (26\%). 235 Health facilities located in Rangpur are least likely to have trained staff for ANC compared to 236 those located in other divisions.

\section{Guidelines on ANC}

238 About $46 \%$ of health facilities providing ANC have reported ANC guidelines available at the 239 facility on the day of interview of the survey. Among all selected covariates, only type of facility 
240 and division have significant association with availability of ANC guidelines. Only $7 \%$ of private 241 hospitals follow ANC guidelines, while about three-fourth of NGO hospitals or clinics (75\%) have 242 reported to have ANC guidelines available. Approximately three in ten of facilities in Sylhet have

243 ANC guidelines, whereas two-third of facilities located in Khulna follows ANC guidelines.

\section{Equipment (blood pressure apparatus)}

245 More than eight in ten of facilities offering ANC have confirmed the availability of functioning 246 digital blood pressure machine or functioning manual sphygmomanometer with stethoscope at the 247 facility. All the covariates except health provider status are significantly associated with the 248 availability of blood pressure apparatus. About $97 \%$ of urban facilities have functioning blood 249 pressure apparatus as compared with $86 \%$ of rural health facilities. Community clinics are least 250 likely (83\%) to avail functioning blood pressure apparatus compared to other facility types. More 251 than nine in ten of facilities who have specialist or MBBS doctors as health providers have reported 252 blood pressure apparatus available for antenatal care as compared with $85 \%$ of facilities who have 253 other health professionals. With increasing basic amenities, the availability of blood pressure 254 apparatus increases from $79 \%$ of facilities with inadequate amenities to $92 \%$ of those with 255 adequate amenities. Health facilities of Barisal are least likely (80\%) to have functioning blood 256 pressure machine than those of other divisions.

\section{Diagnostic capacity (hemoglobin test and urine protein test)}

258 Only $14 \%$ of health facilities giving ANC services have reported functioning equipment and 259 reagents needed to conduct diagnostic tests for ANC (hemoglobin test and urine protein test) 260 available at the facility. All covariates but health provider status have significant association with 261 the capacity to run tests for hemoglobin and urine protein. Only $9 \%$ of rural facilities have 
262 available equipment and reagents for hemoglobin and urine protein tests as compared with around

263 three-fourth of urban facilities. About $6 \%$ of Community clinics and $17 \%$ of public hospitals or

264 clinics have the capacity for hemoglobin and urine protein tests, whereas more than $70 \%$ of NGO

265 hospitals/clinics as well as private hospitals are capable to conduct these tests. Facilities with

266 specialist or MBBS doctors $(67 \%)$ are almost 7.5 times as likely as those with other health

267 providers $(9 \%)$ to conduct these tests. Again, facilities with adequate basic amenities are three

268 times as likely as those with inadequate amenities to perform these tests. Facilities of Rangpur are

269 least likely $(5 \%)$ to conduct hemoglobin and urine protein tests compared to those of other

270 divisions.

\section{Medicines (iron and/or folic acid tablets)}

272 Majority of health facilities that offer ANC have essential medicines (iron and/or folic acid tablets)

273 for ANC. Each selected covariate has been observed to have significant association with 274 availability of these medicines needed for ANC. About $88 \%$ of urban facilities have iron 275 supplements and/or folic acid tablet as compared with 93\% of rural facilities. Private hospitals are 276 least likely $(78 \%)$ to have these essential medicines than other facility types. About $80 \%$ of 277 facilities not having assigned health providers have these two medicines, whereas $93 \%$ of those 278 having assigned health providers reported to have these medicines available at the facility. 279 Approximately nine in ten of facilities with specialist or MBBS doctors have these medicines, 280 while $93 \%$ of facilities who have other health professionals have confirmed these medicines 281 available for antenatal care. The availability of iron and/or folic acid tablets increases as basic 282 amenities index increases. Facilities of Chattogram (83\%) and Sylhet (85\%) are less likely to have 283 these medicines available than facilities of other divisions. 


\section{Multinomial logistic results}

287 The covariates found to be significantly associated with readiness on antenatal care services in

288 bivariate analysis are selected for multivariate analysis. In this study, multinomial logistic

289 regression analysis has been performed considering low readiness as baseline outcome category

290 for the comparison with medium readiness and high readiness. Table 3 shows the coefficients,

291 relative risk ratios (RRR) with 95\% confidence intervals, p-values obtained from analysis.

\section{Medium versus low readiness}

293 Table 3 reveals that the urban and rural facilities have the equal risk for being moderately ready 294 relative to lower ready to provide the ANC services [RRR 1.05; 95\% CI: 0.56-1.99; p-value 0.880].

295 The relative risk of NGO hospitals or clinics are 4.68 times compared to public hospitals or clinics 296 to provide average quality of antenatal care services [RRR 4.68; 95\% CI: 2.54-8.62; p<0.001]. By 297 contrast, community clinics and private hospitals have 31\% [RRR 0.69; 95\% CI: 0.47-0.99, p298 value 0.048 ] and 88\% [RRR 0.12; 95\% CI: 0.05-0.27; $\mathrm{p}<0.001]$ lower probability to be medium 299 ready to offer ANC compared to public hospitals or clinics, respectively. The relative risk of being 300 average ready relative to lower ready for ANC service would be expected to increase by a factor 301 of 4.06 among the facilities with qualified physician i.e., specialist or MBBS doctors as health 302 providers than those with other health providers [RRR 4.02; 95\% CI: 2.24-7.21, p<0.001]. Again, 303 the expected risks of having average readiness are increased by 2.54 [RRR 2.54; 95\% CI: $1.52-$ $3044.26, \mathrm{p}<0.001]$ and 1.70 [RRR 1.01; 95\% CI: 1.01-2.88, p-value 0.047] for those facilities which 305 have moderate and adequate basic amenities, respectively compared to the facilities with 306 inadequate basic amenities. Facilities of Chattogram, Khulna and Mymensingh are significantly 
associated with the increased probability of providing medium quality of ANC services compared to facilities located in Dhaka. Location of facility has no significant effect on medium readiness vs. low readiness to deliver ANC services.

\section{Complete versus low readiness}

Table 3 also demonstrates the results of the comparison between high and low readiness for delivering antenatal care services. The fact that a facility is located in rural area reduces the risk of being complete readiness relative to low readiness to provide the services by a factor of 0.13 compared to an urban facility [RRR $0.13 ; 95 \%$ CI: $0.06-0.31, \mathrm{p}<0.001]$. Community clinics and private hospitals also decrease the probabilities for getting the quality ANC services by the factor of 0.15 and 0.02 , respectively compared to public hospital or clinics. On the other hand, the relative risk of NGO hospitals or clinics are 2.63 times compared to public hospitals or clinics to provide full quality of antenatal care services [RRR 2.63; 95\% CI: 1.27-5.46; p-value 0.010]. The chance of giving quality ANC services increases by a factor of 2.22 when a facility has MBBS doctor or specialist health provider [RRR 2.22; 95\% CI: $0.97-5.08$, p-value 0.058] and it is noted that the evidence is justified at $10 \%$ level of significance. Compared to the facilities having inadequate basic amenities, the expected risk of giving quality ANC services are found to be equal among the facilities having average or adequate basic amenities. Facilities of all divisions except Rangpur are significantly associated with increased chance of getting higher readiness to offer ANC services compared to facilities of Dhaka.

\section{Discussion}

The aim of the present study is to evaluate the readiness of health facilities to provide proper antenatal care (ANC) services among pregnant women and also to determine its associated factors 
using data from the National Survey of Bangladesh. To our knowledge, this is the first study to assess the readiness for offering ANC services in Bangladesh following WHO recommendation regarding service readiness indicators using a nationally representative health facility survey, where the health facilities were considered as sampling units. However, the survey found a huge quality gap for providing ANC services. Most facilities (72.11\%) are poorly prepared, i.e., the indicators for ANC services are not adequately available to provide ANC services, where only $4.26 \%$ are fully prepared to provide. Again, compared with other indicators of ANC, diagnostic capacity (hemoglobin and urine tests) was not found in most facilities (13.66\%), although diagnosis is required at this stage of pregnancy for better health of women as well as their preterm infants. The lack of WHO-recommended indicators for ANC in any facility may refrain women from visiting four or more ANCs as the previous studies have shown that optimal ANC coverage often depends on the availability, cost-effectiveness, and quality of ANC services [35-38]. It is noted that only $47 \%$ of women received healthcare four or more times from providers during their pregnancy in Bangladesh [18]. Nevertheless, the equipment (blood pressure apparatus) and drugs (iron or folic acid tablets) for ANC are now in a better position in terms of availability in Bangladesh than other proposed requirements. Findings related to the readiness of health facilities for quality ANC services are consistent with the studies in Nepal and Ethiopia that the health facilities did not meet the requirements set by WHO guidelines $[8,17]$. Again, another study conducted in India also measured the low quality of ANC services, although the study defined the quality of ANC services based on clinical quality, interpersonal quality of care and utilization of ANC measures [39]. A study on quality ANC readiness in sub-Saharan Africa found out the readiness in specific tracer indicators of ANC services, where most of the countries selected in the study had better readiness than Bangladesh in availability of trained staff, ANC guidelines and 
352 Iron supplementation [16]. In addition, compared to previous survey of 2014 BHFS, the present

353 study did not find any considerable change in preparedness of the health facilities to provide ANC

354 services [40].

\section{(Table 3 is here)}

356 This study also found some potential factors responsible for lower readiness of health facilities to 357 offer ANC services. In the study, the outcome variable "readiness index" have three categories, 358 and lower readiness was considered as the reference (or base) category for both medium and high 359 readiness to run the multinomial logistic regression model. The urban and rural location of the 360 facilities do not have significant difference in medium readiness but exist difference in high 361 readiness, and rural facilities are less likely to be ready fully for providing ANC services compared 362 to urban facilities. Several studies found the similar findings, where the studies explored the 363 general services readiness, and explained the reasons behind the finding that ineffective coverage 364 of health facilities in rural facilities and tertiary or highest-level care in urban facilities [22, 41365 44]. Usually, the qualified health professionals are available to provide maternal health services in 366 most urban facilities compared to rural facilities [18, 45], and according to their advice to their 367 patients regarding the hemoglobin test, urine test, blood pressure apparatus and taking of iron or 368 folic acids tablets, the authorities of urban facilities try to provide these necessary ANC services 369 in the facilities. Again, the discrimination between urban and rural facilities was also observed in 370 bivariate analysis for the above services (Table 2). There may have another reason behind this 371 difference that comparatively the urban people are educated and more concerned about their 372 health, and naturally, they will try to get the quality services from the health facilities which 373 indirectly may influence the managing authorities in urban facilities to provide quality ANC 374 services. Again, The finding of medium readiness versus low readiness was consistent with a 
375 recent study in Ethiopia [8], where the study did not find any significant difference by location of 376 facilities in providing ANC services. The reverse situation was observed in Burkina Faso, Ghana 377 and Tanzania that the rural health facilities had a satisfactory level of quality ANC service [46].

378 The facility types significantly influence the preparedness of the facilities for providing ANC 379 service, and the readiness is better in NGO hospitals or clinics but it is not good in private hospitals 380 or community clinics compared to public hospitals or clinics. Similar studies were found in 381 Ethiopia, Vietnam, and India, where the studies revealed that public health facilities were better in 382 readiness than the private facilities to provide ANC service [8, 47, 48]. The finding was also 383 consistent with a study of 46 low and middle-income countries, and the study revealed that public 384 facilities are the primary source of getting ANC services in a country [8, 49]. This might be 385 happened due to the reason that the government health monitoring team may routinely monitor the 386 public health facilities for ensuring quality health services, and the Directorate General of Health 387 Services (DGHS) has already taken the initiative of measuring performance, ranking, and 388 rewarding the different tiers of health facilities in 2014 for improvement of health services in the 389 public health sector. Again, the opposite scenario of providing the services was observed in NGO 390 facilities. NGO hospitals or clinics in local areas are mainly functioned to help the poor local 391 people and run by financial assistance from the Government of Bangladesh and foreign donors. 392 Many NGOs have succeeded in providing child and maternal health services to their clients. In 393 addition, USAID and other international organizations provide grants and funds to NGOs to 394 provide quality health services, and also the organizations monitor the NGO facilities to strictly 395 follow the guidelines [22, 50].

396 The preparation of health facilities for the delivery of quality ANC services also depends on the 397 qualifications of the health provider. The facilities having qualified physicians, such as specialists 
or MBBS doctors, are more likely to be considered as ready to provide ANC services than other

399 facilities. Recently, the WHO has recommended eight or more health check-ups on women during

400 pregnancy by qualified health professionals in healthcare to reduce pregnancy-related

401 complications that result in death in the mother or their newborn [15]. Thus, the qualifications of

402 a health provider play an important role in providing quality healthcare in any facility. Eligible

403 physicians are always up to date on any guidelines related to health issues and they may be able to

404 persuade managing authorities of the facilities to strictly follow WHO guidelines regarding the

405 availability of ANC service indicators.

406 The basic amenities of health facilities significantly associated with medium readiness but it did

407 not affect the high readiness to provide ANC services. Investigations prove that it is not always

408 true that well-equipped healthcare provides quality ANC services, as there is no difference among

409 inadequate, moderate and adequately equipped facilities in quality services. For example, private

410 hospitals are generally equipped with more basic amenities than public hospitals [2, 22], but our

411 research has shown that private hospitals are less prepared to provide quality ANC services. But

412 this study suggests that health facilities should have adequate basic facilities in addition to

413 maintaining the quality of service.

414 A significant association among division and readiness index was observed in the study, where the

415 health facilities from Chattogram, Khulna and Mymensingh regions were more likely to have

416 average or complete readiness to provide the ANC service compared to Dhaka region, but the

417 facilities from Rangpur and Dhaka regions were equally likely to have medium or complete

418 readiness for providing the service. Again, the facilities from Barisal, Rajshahi and Rangpur

419 regions had the higher risk of being completely ready, but these facilities had no enough evidence

420 to exist the difference related to medium readiness with the facilities in Dhaka region. This can 
421 happen due to regional variation in providing health services or access to better health facilities

422 [28]. Similar results were also found in several studies for quality ANC services [8, 49].

423 Limitations

424 This study focused only on the preparedness of health facilities to provide ANC services for 425 intervention, but didn't provide a guarantee for ensuring quality ANC services. Because, to ensure 426 quality services, we also need to include the followings: health provider knowledge, provider

427 effort, motivation, supervision or increased workloads $[16,51]$ which are not available in the SPA 428 survey. Another limitation is the arbitrary use of cut-off points (below 75\%, 75-99\%, and 100\%)

429 to define the categories of outcome variable "readiness index" that may incorrectly classify the 430 readiness for ANC service delivery. Again, the causal relationship of the factors with readiness of 431 health facilities cannot be examined cause, as the BHFS or SPA data was in a cross-sectional setup.

432 Moreover, the lack of previous studies related to the readiness of health facilities for the provision 433 of ANC services using SPA or HFS data has made it difficult to compare the results of the current 434 study with those of other studies.

435 Conclusion

436 The quality of ANC services is extremely poor in Bangladesh. So, in order to provide quality ANC 437 services, it is necessary to accelerate the availability of several ANC service indicators. Reducing 438 the gap in quality ANC services can be a positive aspect in reaching SDGs related to maternity 439 and neonatal mortality [9, 17, 52, 53]. Again, to ensure the readiness among the facilities for 440 improving quality, routine and robust monitoring in health facilities are required [17, 54]. The 441 Ministry of Health and Family Welfare (MOHFW) is in charge of providing primary healthcare in 442 rural areas through various channels. Among the channels, the community clinics and subdistrict 
443 health complexes play a major role to provide quality healthcare services in rural areas. Therefore, 444 the MOHFW should take the responsibility for ensuring quality ANC services in rural areas by

445 improving and monitoring the community clinics and subdistrict health complexes. This study 446 recommends that the government of Bangladesh should take the initiative of engaging qualified 447 health professionals and arranging routine monitoring in rural health facilities for getting quality 448 health services. In addition, the DGHS should offer incentives to private health facilities to follow 449 the provision and integration of ANC services.

450 Abbreviations

451 ANC: Antenatal Care; WHO: World Health Organization; HFS: Health Facility Survey; BHFS: 452 Bangladesh Health Facility Survey; MMR: Maternal Mortality Ratio; NMR: Neonatal Mortality 453 Rate; SDG: Sustainable Development Goal; HPNSDP: Health, Population and Nutrition Sector 454 Development Program; BDHS: Bangladesh Demographic and Health Survey; SPA: Service 455 Provision Assessment; FP: Family Planning; NIPORT: National Institute of Population Research 456 and Training; MOHFW: Ministry of Health and Family Welfare; USAID: United States Agency 457 for International Development; SARA: Service Availability and Readiness Assessment; NGO: 458 Non-Governmental Organization; MBBS: Bachelor of Medicine, Bachelor of Surgery; PC: 459 Principal Component; RRR: Relative Risk Ratio; CI: Confidence Interval; DGHS: Directorate 460 General of Health Services.

461 Acknowledgements

462 The authors would like to thank National Institute of Population Research and Training (NIPORT), 463 Bangladesh for allowing to use the data sets for analysis. 


\section{Funding}

466 Not applicable

467 Availability of data and materials

468 This study entails a secondary analysis of data from 2017 BHFS. The original survey dataset was 469 available online at https://dhsprogram.com/data and the data set was freely downloadable after 470 completing a registration at the website of DHS program.

\section{Authors' contributions}

472 KKS and SN conceptualized and initiated the research question. FTZ and SN carried out the 473 literature review. KKS and SN conducted the statistical analysis. All authors contributed equally 474 in drafting the manuscript. WB supervised the entire study, reviewed the manuscript and 475 incorporated changes as applicable. FTZ and WB finalized latest version. All authors read and 476 approved the final manuscript.

\section{Ethics approval and consent to participate}

478 The survey used a freely available secondary survey data set (BHFS, 2017) which is available 479 online (https://dhsprogram.com/data). The survey was approved by the Ethics Committee of ICF 480 Macro in Calverton, USA, and by the National Institute of Population Research and Training

481 (NIPORT) in Bangladesh, and the authority taken a permission from the participants i.e., facility 482 in charge to participate in the survey (2017), and all participants signed in a consent form. Thus, 483 ethical approval and consent to participate were automatically deemed unnecessary for the current 484 study.

\section{Consent for publication}

486 Not applicable 


\section{Competing interests}

The authors declare that they have no competing interests.

\section{References}

1. Khan KS, Wojdyla D, Say L, Gülmezoglu AM, Van Look PF. WHO analysis of causes of maternal death: a systematic review. The lancet. 2006;367(9516):1066-74.

2. National Institute of Population Research and Training (NIPORT) and ICF. 2019. Bangladesh Health Facility Survey 2017. Dhaka, Bangladesh: NIPORT, ACPR, and ICF.

3. Abate TM, Abeba A. Evaluation of the quality of antenatal care (ANC) service at higher 2 health center in Jimma, South West Ethiopia. Open Access Library Journal. 2015;2(04):1.

4. Lincetto O, Mothebesoane-Anoh S, Gomez P, Munjanja S. Antenatal care. Opportunities for Africa's newborns: Practical data, policy and programmatic support for newborn care in Africa. 2006:55-62.

5. Chukwuma A, Wosu AC, Mbachu C, Weze K. Quality of antenatal care predicts retention in skilled birth attendance: a multilevel analysis of 28 African countries. BMC pregnancy and childbirth. 2017;17:1-10.

6. Adjiwanou V, LeGrand T. Does antenatal care matter in the use of skilled birth attendance in rural Africa: a multi-country analysis. Social science \& medicine. 2013;86:26-34.

7. Afulani PA, Moyer C. Explaining disparities in use of skilled birth attendants in developing countries: a conceptual framework. PLoS One. 2016;11(4):e0154110.

8. Defar A, Getachew T, Taye G, Tadele T, Getnet M, Shumet T, et al. Quality antenatal care services delivery at health facilities of Ethiopia, assessment of the structure/input of care setting. BMC Health Services Research. 2020;20:1-9.

9. Jo Y, Alland K, Ali H, Mehra S, LeFevre AE, Pak SE, et al. Antenatal care in rural Bangladesh: current state of costs, content and recommendations for effective service delivery. BMC Health Services Research. 2019;19:1-13.

10. United Nations Development Program. Sustainable development goals - United Nations. 2016. https://www.un.org/sustainabledevelopment/health/. Accessed 29 March 2021.

11. World Health Organization. Newborns: improving survival and well-being. 2020. https://www.who.int/news-room/fact-sheets/detail/newborns-reducing-mortality. Accessed 29 March 2021.

12. World Health Organization. Maternal Mortality. 2019. https://www.who.int/newsroom/fact-sheets/detail/maternal-mortality. Accessed 29 March 2021.

13. World Bank. The millennium development goals for health: rising to the challenges. 2004. https://elibrary.worldbank.org/doi/abs/10.1596/0-8213-5767-0.

14. Tinker A. Women's health: the unfinished agenda. International Journal of Gynecology \& Obstetrics. 2000;70(1):149-58.

15. World Health Organization (WHO). WHO recommendations on antenatal care for a positive $\quad$ pregnancy 2016. https://www.who.int/publications/i/item/9789241549912. Accessed 29 March 2021. 
16. Kanyangarara M, Munos MK, Walker N. Quality of antenatal care service provision in health facilities across sub-Saharan Africa: Evidence from nationally representative health facility assessments. Journal of global health. 2017;7(2).

17. Lama TP, Munos MK, Katz J, Khatry SK, LeClerq SC, Mullany LC. Assessment of facility and health worker readiness to provide quality antenatal, intrapartum and postpartum care in rural Southern Nepal. BMC Health Services Research. 2020;20:1-12.

18. National Institute of Population Research and Training (NIPORT), and ICF. 2020. Bangladesh Demographic and Health Survey 2017-18. Dhaka, Bangladesh, and Rockville, Maryland, USA: NIPORT and ICF.

19. Afulani PA, Buback L, Essandoh F, Kinyua J, Kirumbi L, Cohen CR. Quality of antenatal care and associated factors in a rural county in Kenya: an assessment of service provision and experience dimensions. BMC Health Services Research. 2019;19:1-16.

20. Afulani PA, Kirumbi L, Lyndon A. What makes or mars the facility-based childbirth experience: thematic analysis of women's childbirth experiences in western Kenya. Reproductive health. 2017;14:1-13.

21. Obago IT. The role of antenatal care in predicting health facility delivery among women in Kenya: further analysis of data from the 2008-09 KDHS: ICF International; 2013.

22. Yusuf SS, Acharya K, Ahmed R, Ahmed A. Understanding general health service readiness and its correlates in the health facilities of Bangladesh: evidence from the Bangladesh Health Facility Survey 2017. Journal of Public Health. 2021:1-12.

23. Seiglie JA, Serván-Mori E, Begum T, Meigs JB, Wexler DJ, Wirtz VJ. Predictors of health facility readiness for diabetes service delivery in low-and middle-income countries: The case of Bangladesh. Diabetes research and clinical practice. 2020;169:108417.

24. Alam W, Nujhat S, Parajuli A, Banyira L, Mohsen WAM, Sutradhar I, et al. Readiness of primary health-care facilities for the management of non-communicable diseases in rural Bangladesh: a mixed methods study. The Lancet Global Health. 2020;8:S17.

25. Islam MR, Laskar SP, Macer D. A study on service availability and readiness assessment of non-communicable diseases using the who tool for Gazipur district in Bangladesh. Bangladesh Journal of Bioethics. 2016;7(2):1-13.

26. Paromita P, Chowdhury HA, Mayaboti CA, Rakhshanda S, Rahman AF, Karim MR, et al. Assessing service availability and readiness to manage Chronic Respiratory Diseases (CRDs) in Bangladesh. PLoS One. 2021;16(3):e0247700.

27. Biswas T, Haider MM, Gupta RD, Uddin J. Assessing the readiness of health facilities for diabetes and cardiovascular services in Bangladesh: a cross-sectional survey. BMJ open. 2018;8(10):e022817.

28. Sen KK, Nilima S, Khokan MR. Readiness of Health Facilities to Provide Child Curative Care Services: Evidence Based on Bangladesh Health Facility Survey, 2014. The Dhaka University Journal of Science. 2020;68(1):29-35.

29. Shawon MSR, Adhikary G, Ali MW, Shamsuzzaman M, Ahmed S, Alam N, et al. General service and child immunization-specific readiness assessment of healthcare facilities in two selected divisions in Bangladesh. BMC Health Services Research. 2018;18:1-10.

30. World Health Organization. Service availability and readiness assessment (SARA): an annual monitoring system for service delivery: reference manual. 2013. https://www.who.int/healthinfo/systems/SARA Reference Manual Full.pdf. 
31. Leslie HH, Spiegelman D, Zhou X, Kruk ME. Service readiness of health facilities in Bangladesh, Haiti, Kenya, Malawi, Namibia, Nepal, Rwanda, Senegal, Uganda and the United Republic of Tanzania. Bulletin of the World Health Organization. 2017;95(11):738.

32. Bintabara D, Nakamura K, Seino K. Determinants of facility readiness for integration of family planning with HIV testing and counseling services: evidence from the Tanzania service provision assessment survey, 2014-2015. BMC Health Services Research. 2017;17:1-11.

33. Acharya K, Thapa R, Bhattarai N, Bam K, Shrestha B. Availability and readiness to provide sexually transmitted infections and HIV testing and counselling services in Nepal: evidence from comprehensive health facility survey. BMJ open. 2020;10(12):e040918.

34. Hosner DW, Lemeshow S. Applied logistic regression. New York: Jhon Wiley \& Son. 1989.

35. Ali N, Sultana M, Sheikh N, Akram R, Mahumud RA, Asaduzzaman M, et al. Predictors of optimal antenatal care service utilization among adolescents and adult women in Bangladesh. Health services research and managerial epidemiology. 2018;5:2333392818781729.

36. Chakraborty N, Islam MA, Chowdhury RI, Bari W. Utilisation of postnatal care in Bangladesh: evidence from a longitudinal study. Health \& social care in the community. 2002;10(6):492-502.

37. Kabir M, Iliyasu Z, Abubakar I, Sani A. Determinants of utilization of antenatal care services in Kumbotso village, Northern Nigeria. Tropical doctor. 2005;35(2):110-.

38. Kulmala T, Vaahtera M, Rannikko J, Ndekha M, Cullinan T, SALIN ML, et al. The relationship between antenatal risk characteristics, place of delivery and adverse delivery outcome in rural Malawi. Acta Obstetricia et Gynecologica Scandinavica: ORIGINAL ARTICLE. 2000;79(11):984-90.

39. Rani M, Bonu S, Harvey S. Differentials in the quality of antenatal care in India. International journal for quality in health care. 2008;20(1):62-71.

40. National Institute of Population Research and Training (NIPORT), Associates for Community and Population Research (ACPR), and ICF International. 2016. Bangladesh Health Facility Survey 2014. Dhaka, Bangladesh: NIPORT, ACPR, and ICF International. .

41. Wang W, Mallick L, Allen C, Pullum T. Effective coverage of facility delivery in Bangladesh, Haiti, Malawi, Nepal, Senegal, and Tanzania. PLoS One. 2019;14(6):e0217853.

42. Gage AJ, Ilombu O, Akinyemi Al. Service readiness, health facility management practices, and delivery care utilization in five states of Nigeria: a cross-sectional analysis. BMC pregnancy and childbirth. 2016;16:1-13.

43. Acharya K, Paudel YR. General health service readiness and its association with the facility level indicators among primary health care centers and hospitals in Nepal. Journal of Global Health Reports. 2019;3.

44. Tecla SJ, Franklin B, David A, Jackson TK. Assessing facility readiness to offer basic emergency obstetrics and neonatal care (BEmONC) services in health care facilities of west Pokot county, Kenya. J Clin Simul Res. 2017;7:25-39.

45. National Institute of Population Research and Training (NIPORT), Mitra and Associates, and ICF International. 2016. Bangladesh Demographic and Health Survey 2014. Dhaka, Bangladesh, and Rockville, Maryland, USA: NIPORT, Mitra and Associates, and ICF International. . 
46. Duysburgh E, Zhang WH, Ye M, Williams A, Massawe S, Sié A, et al. Quality of antenatal and childbirth care in selected rural health facilities in Burkina Faso, Ghana and Tanzania: similar finding. Tropical medicine \& international health. 2013;18(5):534-47.

47. Tuan T, Dung VTM, Neu I, Dibley MJ. Comparative quality of private and public health services in rural Vietnam. Health Policy and Planning. 2005;20(5):319-27.

48. Mundodan JM. Structural quality of antenatal clinics in the public health sector in a northern district in Kerala, India. JM al Int J Community Med Public Heal. 2015;2(4):5139.

49. Powell-Jackson T, Macleod D, Benova L, Lynch C, Campbell OM. The role of the private sector in the provision of antenatal care: a study of Demographic and Health Surveys from 46 low-and middle-income countries. Tropical medicine \& international health. 2015;20(2):230-9.

50. Lance P, Angeles G, Kamal N. Bangladesh Smiling Sun Franchise Program (BSSFP): Impact Evaluation Report: Measure 2012. https://www.measureevaluation.org/resources/publications/tr-1289/at download/document. Accessed 23 January 2020.

51. Baker U, Peterson S, Marchant T, Mbaruku G, Temu S, Manzi F, et al. Identifying implementation bottlenecks for maternal and newborn health interventions in rural districts of the United Republic of Tanzania. Bulletin of the World Health Organization. 2015;93:380-9.

52. Koblinsky M, Moyer CA, Calvert C, Campbell J, Campbell OM, Feigl AB, et al. Quality maternity care for every woman, everywhere: a call to action. The lancet. 2016;388(10057):2307-20.

53. Kinney MV, Kerber KJ, Black RE, Cohen B, Nkrumah F, Coovadia H, et al. Sub-Saharan Africa's mothers, newborns, and children: where and why do they die? PLoS Med. 2010;7(6):e1000294.

54. Shankar A, Bartlett L, Fauveau V, Islam M, Terreri N. Delivery of MDG 5 by active management with data. Lancet (London, England). 2008;371(9620):1223-4. 
